\section{Party on!}

A New, Conditional Variable-Importance Measure for Random Forests Available in the party Package

\section{by Carolin Strobl, Torsten Hothorn and Achim Zeileis}

\begin{abstract}
Random forests are one of the most popular statistical learning algorithms, and a variety of methods for fitting random forests and related recursive partitioning approaches is available in $\mathrm{R}$. This paper points out two important features of the random forest implementation cforest available in the party package: The resulting forests are unbiased and thus preferable to the randomForest implementation available in randomForest if predictor variables are of different types. Moreover, a conditional permutation importance measure has recently been added to the party package, which can help evaluate the importance of correlated predictor variables. The rationale of this new measure is illustrated and hands-on advice is given for the usage of recursive partitioning tools in $\mathrm{R}$.
\end{abstract}

Recursive partitioning methods are amongst the most popular and widely used statistical learning tools for nonparametric regression and classification. Random forests in particular, which can deal with large numbers of predictor variables even in the presence of complex interactions, are being applied successfully in many scientific fields (see, e.g., Lunetta et al., 2004; Strobl et al., 2009, and the references therein for applications in genetics and social sciences). Thus, it is not surprising that there is a variety of recursive partitioning tools available in $\mathrm{R}$ (see http://CRAN.R-project.org/view= MachineLearning for an overview).

The scope of recursive partitioning methods in $\mathrm{R}$ ranges from the standard classification and regression trees available in rpart (Therneau et al., 2008) to the reference implementation of random forests (Breiman, 2001) available in randomForest (Liaw and Wiener, 2002, 2008). Both methods are popular in applied research, and several extensions and refinements have been suggested in the statistical literature in recent years.

One particularly important improvement was the introduction of unbiased tree algorithms, which overcome the major weak spot of the classical approaches available in rpart and randomForest: variable-selection bias. The term variable-selection bias refers to the fact that in standard tree algorithms variable selection is biased in favor of variables offering many potential cut-points, so that variables with many categories and continuous variables are artificially preferred (see, e.g, Kim and Loh, 2001; Shih, 2002; Hothorn et al., 2006; Strobl et al., 2007a, for de- tails).

To overcome this weakness of the early tree algorithms, new algorithms have been developed that do not artificially favor splits in variables with many categories or continuous variables. In $\mathrm{R}$ such an unbiased tree algorithm is available in the ctree function for conditional inference trees in the party package (Hothorn et al., 2006). The package also provides a random forest implementation cforest based on unbiased trees, which enables learning unbiased forests (Strobl et al., 2007b).

Unbiased variable selection is the key to reliable prediction and interpretability in both individual trees and forests. However, while a single tree's interpretation is straightforward, in random forests an extra effort is necessary to assess the importance of each predictor in the complex ensemble of trees.

This issue is typically addressed by means of variable-importance measures such as Gini importance and the "mean decrease in accuracy" or "permutation" importance, available in randomForest in the importance() function (with type $=2$ and type $=1$, respectively). Similarly, a permutationimportance measure for cforest is available via varimp () in party.

Unfortunately, variable-importance measures in random forests are subject to the same bias in favor of variables with many categories and continuous variables that affects variable selection in single trees, and also to a new source of bias induced by the resampling scheme (Strobl et al., 2007b). Both problems can be addressed in party to guarantee unbiased variable selection and variable importance for predictor variables of different types.

Even though this refined approach can provide reliable variable-importance measures in many applications, the original permutation importance can be misleading in the case of correlated predictors. Therefore, Strobl et al. (2008) suggested a solution for this problem in the form of a new, conditional permutation-importance measure. Starting from version 0.9-994, this new measure is available in the party package.

The rationale and usage of this new measure is outlined in the following sections and illustrated by means of a toy example.

\section{Random forest variable-importance measures}

Permutation importance, which is available in randomForest and party, is based on a random permutation of the predictor variables, as described in more detail below.

The alternative variable-importance measure 
available in randomForest, Gini importance, is based on the Gini gain criterion employed in most traditional classification tree algorithms. However, the Gini importance has been shown to carry forward the bias of the underlying Gini-gain splitting criterion (see, e.g., Kim and Loh, 2001; Strobl et al., 2007a; Hothorn et al., 2006) when predictor variables vary in their number of categories or scale of measurement (Strobl et al., 2007b). Therefore, it is not recommended in these situations.

Permutation importance, on the other hand, is a reliable measure of variable importance for uncorrelated predictors when sub-sampling without replacement — instead of bootstrap sampling - and unbiased trees are used in the construction of the forest (Strobl et al., 2007b). Accordingly, the default settings for the control parameters cforest_control have been pre-defined to the default version cforest_unbiased to guarantee subsampling without replacement and unbiased individual trees in fitting random forests with the party package.

The rationale of the original permutationimportance measure is the following: By randomly permuting the predictor variable $X_{j}$, its original association with the response $Y$ is broken. When the permuted variable $X_{j}$, together with the remaining non-permuted predictor variables, is used to predict the response for the out-of-bag observations, the prediction accuracy (i.e., the number of correctly classified observations in classification, or respectively the mean squared error in regression) decreases substantially if the original variable $X_{j}$ was associated with the response. Thus, Breiman (2001) suggests the difference in prediction accuracy before and after permuting $X_{j}$, averaged over all trees, as a measure for variable importance.

In standard implementations of random forests, such as randomForest in $\mathrm{R}$, an additional scaled version of permutation importance (often called the $z$ score), which is computed by dividing the raw importance by its standard error, is provided (for example by importance (obj, type $=2$, scale = TRUE) in randomForest). Note, however, that the results of Strobl and Zeileis (2008) show that the z-score is not suitable for significance tests and that raw importance has better statistical properties.

\section{Why conditional importance?}

The original permutation-importance measure can, for reasons outlined below, be considered as a marginal measure of importance. In this sense, it has the same property as, e.g., a marginal correlation coefficient: A variable that has no effect of its own, but is correlated with a relevant predictor variable, can receive a high importance score. Accordingly, empirical results (see Strobl et al., 2008, and the refer- ences therein) suggest that the original permutationimportance measure often assigns higher scores to correlated predictors.

In contrast, partial correlation coefficients (like the coefficients in linear regression models) measure the importance of a variable given the other predictor variables in the model. The advantage of such a partial, or conditional, approach is illustrated by means of a toy example: The data set readingSkills is an artificial data set generated by means of a linear model. The response variable contains hypothetical scores on a test of reading skills for 200 school children. Potential predictor variables in the data set are the age of the child, whether the child is a native speaker of the test language and the shoe size of the child.

Obviously, the latter is not a sensible predictor of reading skills (and was actually simulated not to have any effect on the response) - but with respect to marginal (as opposed to partial) correlations, shoe size is highly correlated with the test score. Of course this spurious correlation is only due to the fact that both shoe size and test score are associated with the underlying variable age.

In this simple problem, a linear model would be perfectly capable of identifying the original coefficients of the predictor variables (including the fact that shoe size has no effect on reading skills once the truly relevant predictor variable age is included in the model). However, the cforest permutationimportance measure is mislead by the spurious correlation and assigns a rather high importance value to the nonsense-variable shoe size:

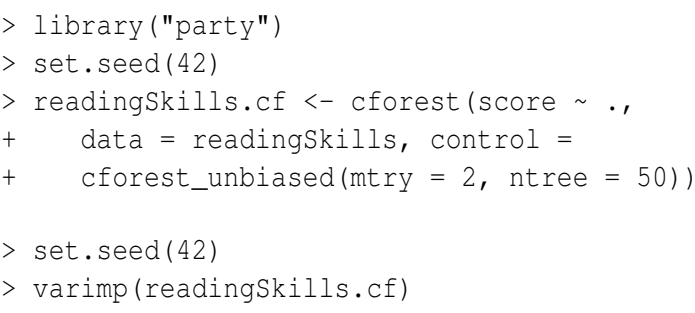

The reason for this odd behavior can be found in the way the predictor variables are permuted in the computation of the importance measure: Strobl et al. (2008) show that the original approach, where one predictor variable $X_{j}$ is permuted against both the response $Y$ and the remaining (one or more) predictor variables $Z=X_{1}, \ldots, X_{j-1}, X_{j+1}, \ldots, X_{p}$, as illustrated in Figure 1, corresponds to a pattern of independence between $X_{j}$ and both $Y$ and $Z$.

From a theoretical point of view, this means that a high value of the importance measure can be caused by a violation either of the independence between $X_{j}$ and $Y$ or of the independence between $X_{j}$ and $Z$, even though the latter is not of interest here. For practical applications, this means that a variable $X_{j}$ 
that is correlated with an important predictor $Z$ can appear more important than an uncorrelated variable, even if $X_{j}$ has no effect of its own.

\begin{tabular}{ccc}
\hline$Y$ & $X_{j}$ & $Z$ \\
\hline$y_{1}$ & $x_{\pi_{j}(1), j}$ & $z_{1}$ \\
$\vdots$ & $\vdots$ & $\vdots$ \\
$y_{i}$ & $x_{\pi_{j}(i), j}$ & $z_{i}$ \\
$\vdots$ & $\vdots$ & $\vdots$ \\
$y_{n}$ & $x_{\pi_{j}(n), j}$ & $z_{n}$ \\
\hline
\end{tabular}

Figure 1: Permutation scheme for the original permutation-importance measure.

\begin{tabular}{ccc}
\hline$Y$ & $X_{j}$ & $Z$ \\
\hline$y_{1}$ & $x_{\pi_{j \mid Z=a}(1), j}$ & $z_{1}=a$ \\
$y_{3}$ & $x_{\pi_{j \mid Z=a}(3), j}$ & $z_{3}=a$ \\
$y_{27}$ & $x_{\pi_{j \mid Z=a}(27), j}$ & $z_{27}=a$ \\
\hline$y_{6}$ & $x_{\pi_{j \mid Z=b}(6), j}$ & $z_{6}=b$ \\
$y_{14}$ & $x_{\pi_{j \mid Z=b}(14), j}$ & $z_{14}=b$ \\
$y_{21}$ & $x_{\pi_{j \mid Z=b}(21), j}$ & $z_{21}=b$ \\
$\vdots$ & $\vdots$ & $\vdots$ \\
\hline
\end{tabular}

Figure 2: Permutation scheme for the conditional permutation importance.

The aim to reflect only the impact of $X_{j}$ itself in predicting the response $Y$, rather than its correlations with other predictor variables, can be better achieved by means of a conditional importance measure in the spirit of a partial correlation: We want to measure the association between $X_{j}$ and $Y$ given the correlation structure between $X_{j}$ and the other predictor variables in the data set.

To meet this aim, Strobl et al. (2008) suggest a conditional permutation scheme, where $X_{j}$ is permuted only within groups of observations with $Z=z$ in order to preserve the correlation structure between $X_{j}$ and the other predictor variables, as illustrated in Figure 2.

With this new, conditional permutation scheme, the importance measure is able to reveal the spurious correlation between shoe size and reading skills:

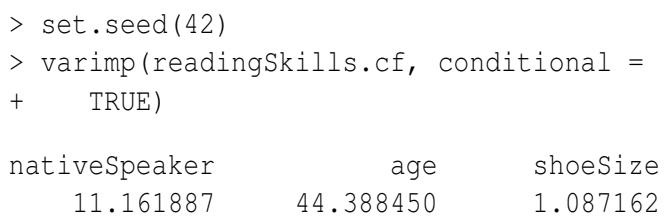

Only by means of conditional importance it becomes clear that the covariate native speaker is actually more relevant for predicting the test score than shoe size is, whose conditional effect is negligible. Thus, the conditional importance mimics the behavior of partial correlations or linear regression coefficients, the interpretation of which many readers may be more familiar. However, whether a marginal or conditional importance measure is to be preferred depends on the actual research question.

Current research also investigates the impact that the choice of the tuning parameter mtry, which regulates the number of randomly preselected predictor variables that can be chosen in each split (cf. Strobl et al., 2008), and parameters regulating the depth of the trees have on variable importance.

\section{How is the conditioning grid de- fined technically?}

Conditioning is straightforward whenever the variables to be conditioned on, $Z$, are categorical (cf., e.g., Nason et al., 2004). However, conditioning on continuous variables, which may entail as many different values as observations in the sample, would produce cells with very sparse counts - which would make permuting the values of $X_{j}$ within each cell rather pointless. Thus, in order to create cells of reasonable size for conditioning, continuous variables need to be discretized.

As a straightforward discretization strategy for random forests, Strobl et al. (2008) suggest defining the conditioning grid by means of the partition of the feature space induced by each individual tree. This grid can be used to conditionally permute the values of $X_{j}$ within cells defined by combinations of $Z$, where $Z$ can contain potentially large sets of covariates of different scales of measurement.

The main advantages of this approach are that this partition has already been learned from the data during model fitting, that it can contain splits in categorical, ordered and continuous predictor variables, and that it can thus serve as an internally available means for discretizing the feature space. For ease of computation, the conditioning grid employed in varimp uses all cut-points as bisectors of the sample space (the same approach is followed by Nason et al., 2004).

The set of variables $Z$ to be conditioned on should contain all variables that are correlated with the current variable of interest $X_{j}$. In the varimp function, this is assured by the small default value 0.2 of the threshold argument: By default, all variables whose correlation with $X_{j}$ meets the condition $1-p$-value $>0.2$ are used for conditioning. A larger value of threshold would have the effect that only those variables that are strongly correlated with $X_{j}$ would be used for conditioning, but would also lower the computational burden.

Note that the same permutation tests that are used for split selection in the tree building process (Hothorn et al., 2006) are used here to measure the association between $X_{j}$ and the remaining covariates. 


\section{A short recipe for fitting random forests and computing variable im- portance measures with $R$}

To conclude, we would like to summarize the application of conditional variable importance and general issues in fitting random forests with R. Depending on certain characteristics of your data set, we suggest the following approaches:

- If all predictor variables are of the same type (for example: all continuous or all unordered categorical with the same number of categories), use either randomForest (randomForest) or cforest (party). While randomForest is computationally faster, cforest is safe even for variables of different types.

For predictor variables of the same type, Gini importance, importance (obj, type $=2$ ), or permutation importance, importance $(\mathrm{obj}$, type =1), available for randomForest, and permutation importance, varimp (obj), available for cforest, are all adequate importance measures.

- If the predictor variables are of different types (for example: different scales of measurement, different numbers of categories), use cforest (party) with the default option controls $=$ cforest_unbiased and permutation importance varimp (obj).

- If the predictor variables are correlated, depending on your research question, conditional importance, available via varimp (obj, conditional = TRUE) for cforest (party), can add to the understanding of your data.

General remarks:

- Note that the default settings for mtry differ in randomforest and cforest: In randomForest the default setting for classification, e.g., is floor(sqrt (ncol (x))), while in cforest it is fixed to the value 5 for technical reasons.

- Always check whether you get the same results with a different random seed before interpreting the variable importance ranking!

If the ranking of even the top-scoring predictor variables depends on the choice of the random seed, increase the number of trees (argument ntree in randomForest and cforest_control).

\section{Bibliography}

L. Breiman. Random forests. Machine Learning, 45(1): 5-32, 2001.

T. Hothorn, K. Hornik, and A. Zeileis. Unbiased recursive partitioning: A conditional inference framework. Journal of Computational and Graphical Statistics, 15(3):651-674, 2006.

H. Kim and W. Loh. Classification trees with unbiased multiway splits. Journal of the American Statistical Association, 96(454):589-604, 2001.

A. Liaw and $\mathrm{M}$. Wiener. Classification and regression by randomForest. R News, 2(3):18-22, 2002.

A. Liaw and M. Wiener. randomForest: Breiman and Cutler's Random Forests for Classification and Regression, 2008. URL http://CRAN.R-project. org/ package=randomForest. $R$ package version 4.5-28.

K. L. Lunetta, L. B. Hayward, J. Segal, and P. V. Eerdewegh. Screening large-scale association study data: Exploiting interactions using random forests. BMC Genetics, 5:32, 2004.

M. Nason, S. Emerson, and M. Leblanc. CARTscans: A tool for visualizing complex models. Journal of Computational and Graphical Statistics, 13(4):1-19, 2004.

Y.-S. Shih. Regression trees with unbiased variable selection. Statistica Sinica, 12:361-386, 2002.

C. Strobl and A. Zeileis. Danger: High power! - Exploring the statistical properties of a test for random forest variable importance. In Proceedings of the 18th International Conference on Computational Statistics, Porto, Portugal, 2008.

C. Strobl, A.-L. Boulesteix, and T. Augustin. Unbiased split selection for classification trees based on the Gini index. Computational Statistics \& Data Analysis, 52(1):483-501, 2007a.

C. Strobl, A.-L. Boulesteix, A. Zeileis, and T. Hothorn. Bias in random forest variable importance measures: Illustrations, sources and a solution. $B M C$ Bioinformatics, 8:25, 2007b.

C. Strobl, A.-L. Boulesteix, T. Kneib, T. Augustin, and A. Zeileis. Conditional variable importance for random forests. BMC Bioinformatics, 9:307, 2008.

C. Strobl, J. Malley, and G. Tutz. An introduction to recursive partitioning: Rationale, application and characteristics of classification and regression trees, bagging and random forests. Psychological Methods, 2009. In press.

T. M. Therneau, B. Atkinson, and B. D. Ripley. rpart: Recursive partitioning. 2008. URL http://CRAN. $\mathrm{R}$-project.org/package=rpart. $\mathrm{R}$ package version 3.1-41.

\section{Carolin Strobl}

Department of Statistics

Ludwig-Maximilians-Universität

Munich, Germany

carolin.stroblestat. uni-muenchen.de 